Supporting Information

\title{
Understanding and Advancing Bifacial Thin Film Solar Cells
}

Adam B. Phillips*, Kamala Khanal Subedi, Geethika K. Liyanage, Fadhil K. Alfadhili, Randy J. Ellingson, and Michael J. Heben

Wright Center for Photovoltaics Innovation and Commercialization, Department of Physics and Astronomy, University of Toledo, Toledo, Ohio, USA 43606

*Corresponding author: Adam.Phillips@UToledo.edu

\section{SCAPs Parameters}

Table S1 shows the material parameters used in the SCAPS -1D simulation, most of which were obtained from previous simulations. ${ }^{1-5}$ The defects were assumed to have Gaussian type energy distribution placed $0.6 \mathrm{eV}$ above the valance band energy. When varying the carrier lifetime, both electron and hole capture cross sections were varied.

Table S1. Material parameters used for SCAPS 1D simulations.

\begin{tabular}{|c|c|c|c|c|}
\hline Parameter & TCO & Emitter-MZO & CdTe & Back-buffer \\
\hline Thickness $(\mathrm{nm})$ & 300 & 100 & 2000 & 100 \\
\hline Band gap (eV) & 3.6 & 3.7 & 1.5 & 3.3 \\
\hline$\chi(\mathrm{eV})$ & 4.45 & 4.2 & 4.4 & 2.4 \\
\hline Relative dielectric constant & 9 & 10 & 9.4 & 9.4 \\
\hline $\mathrm{N}_{c}\left(\mathrm{~cm}^{-3}\right)$ & $2.2 \times 10^{18}$ & $2.2 \times 10^{18}$ & $8.0 \times 10^{17}$ & $8.0 \times 10^{17}$ \\
\hline $\mathrm{N}_{\mathrm{V}}\left(\mathrm{cm}^{-3}\right)$ & $1.8 \times 10^{19}$ & $1.8 \times 10^{19}$ & $1.8 \times 10^{19}$ & $1.8 \times 10^{19}$ \\
\hline Mobility e/h (cm $\left.{ }^{2} / \mathrm{Vs}\right)$ & $50 / 25$ & $100 / 25$ & $320 / 40$ & $320 / 40$ \\
\hline Lifetime e/h (ns) & $0.1 / 0.1$ & $1 / 10^{-3}$ & varying & $0.1 / 0.1$ \\
\hline Doping density $\left(\mathrm{cm}^{-3}\right)$ & $\mathrm{n}: 5 \times 10^{20}$ & $\mathrm{n}: 1 \times 10^{17}$ & $\mathrm{p}: 2 \times 10^{14}-2 \times 10^{16}$ & $\mathrm{p}: 2 \times 10^{14}-1 \times 10^{21}$ \\
\hline Interfaces & TCO/MZO & MzO/CdTe & CdTe/Back-buffer & Back buffer/Electrode \\
\hline $\mathrm{S}_{n}, \mathrm{~S}_{p}(\mathrm{~cm} / \mathrm{s})$ & $\mathrm{N} . \mathrm{A}$. & $10^{5}$ & $10^{0}-10^{4}$ & $10^{5}$ \\
\hline
\end{tabular}

\section{Initial Fermi Level Offset Sign Convention}

The initial Fermi level offset (IFLO) is a difference between the Fermi level of the absorber and the back buffer prior to the two films interacting. The sign convention, shown in Fig. S1, is defined in terms of flow for hole carrier flow, so when the Fermi level for the absorber is deeper for the absorber than back buffer layer, there is no impediment to hole flow and the IFLO is negative. 


\section{CdTe}
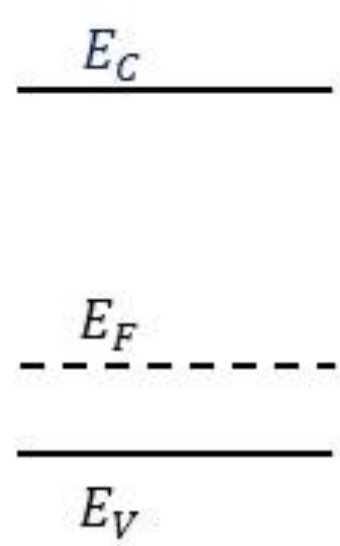

\section{Back}

Buffer

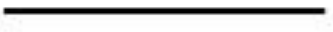

IFLO $<0$

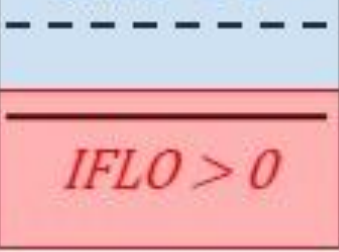

Fig. S1. Non-interacting band diagram of CdTe and the back buffer layer. If the Fermi level of the buffer is above the Fermi level of the absorber (in the blue box, as shown), the initial Fermi level offset (IFLO) is negative. If the Fermi level of the buffer is below the Fermi level of the absorber (in the red box), the IFLO is positive.

\section{Performance of Existing Devices}

As the band diagrams for devices with $4 \mu \mathrm{m}$ and $2 \mu \mathrm{m}$ CdTe absorbers for several bias voltages shown in the main text there are clearly a front and back junction (FJ and BJ, respectively) in the device with a 4 $\mu \mathrm{m}$ thick absorber. Because the depletion region of the BJ extends $\sim 1 \mu \mathrm{m}$ into the device, all of the light with wavelength less than $500 \mathrm{~nm}$ and most of the light from the other wavelengths is absorbed in this region under short circuit conditions, as shown in the zero bias QE measurements shown in Fig. S2. For the $2 \mu \mathrm{m}$ device, the FJ and BJ interact resulting in an equilibrated band diagram with no depletion region at the back of the device at zero bias. Consequently, most of the photogenerated carriers are collected as shown in the QE graph.

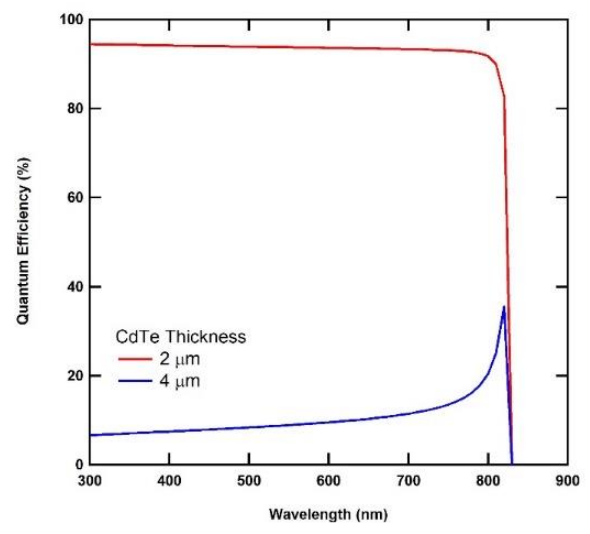

Fig. S2. Simulated quantum efficiency (QE) curves for CdTe devices with varying absorber thickness at short circuit. 


\section{Effect of Back Surface Recombination Velocity}

The PV parameters for CdTe devices illuminated through the front of the device are tabulated in Table S2. These are presented to show how the device improves with decreasing back surface recombination (BSRV).

Table S2. PV parameters of devices illuminated through the front as a function of back surface recombination rate.

\begin{tabular}{|c|c|c|c|c|c|c|c|c|}
\hline & \multicolumn{4}{|c|}{4 microns } & \multicolumn{4}{|c|}{2 microns } \\
\hline $\begin{array}{l}\text { IF Rate } \\
\mathrm{cm} / \mathrm{s}\end{array}$ & $\begin{array}{l}\text { Voc } \\
\mathrm{mV}\end{array}$ & $\begin{array}{c}\text { Jsc } \\
\mathrm{mA} / \mathrm{cm}^{2}\end{array}$ & $\begin{array}{l}\text { FF } \\
\%\end{array}$ & $\begin{array}{l}\text { Eff } \\
\%\end{array}$ & $\begin{array}{l}\text { Voc } \\
\mathrm{mV}\end{array}$ & $\begin{array}{c}\text { Jsc } \\
\mathrm{mA} / \mathrm{cm}^{2}\end{array}$ & $\begin{array}{l}\text { FF } \\
\%\end{array}$ & $\begin{array}{l}\text { Eff } \\
\%\end{array}$ \\
\hline $1 \mathrm{e} 4$ & 837 & 28.5 & 83.0 & 19.8 & 790 & 28.4 & 82.0 & 18.4 \\
\hline $1 \mathrm{e} 3$ & 849 & 28.5 & 82.6 & 19.9 & 826 & 28.4 & 82.4 & 19.3 \\
\hline $1 \mathrm{e} 2$ & 892 & 28.5 & 79.4 & 20.2 & 900 & 28.4 & 78.2 & 20.0 \\
\hline $1 \mathrm{e} 2$ & 917 & 28.5 & 77.7 & 20.3 & 937 & 28.4 & 76.0 & 20.2 \\
\hline $1 \mathrm{e} 1$ & 921 & 28.5 & 77.5 & 20.3 & 942 & 28.4 & 75.6 & 20.2 \\
\hline
\end{tabular}

Fig. S3 shows the simulated current density-voltage and recombination current density-voltage curves as a function of BSRV. These graphs show how the interface recombination decreases, leading to improved device performance. In the case of the $4 \mu \mathrm{m}$ device, the decrease in interface recombination initially leads to increases in short circuit current density, while for the $2 \mu \mathrm{m}$ device, the initial improvements are due to increasing FF. For both devices, SRH begins to affect device performance when BSRV 100 $\mathrm{cm} / \mathrm{s}$ and dominates device performance when BSRV is $10 \mathrm{~cm} / \mathrm{s}$. 

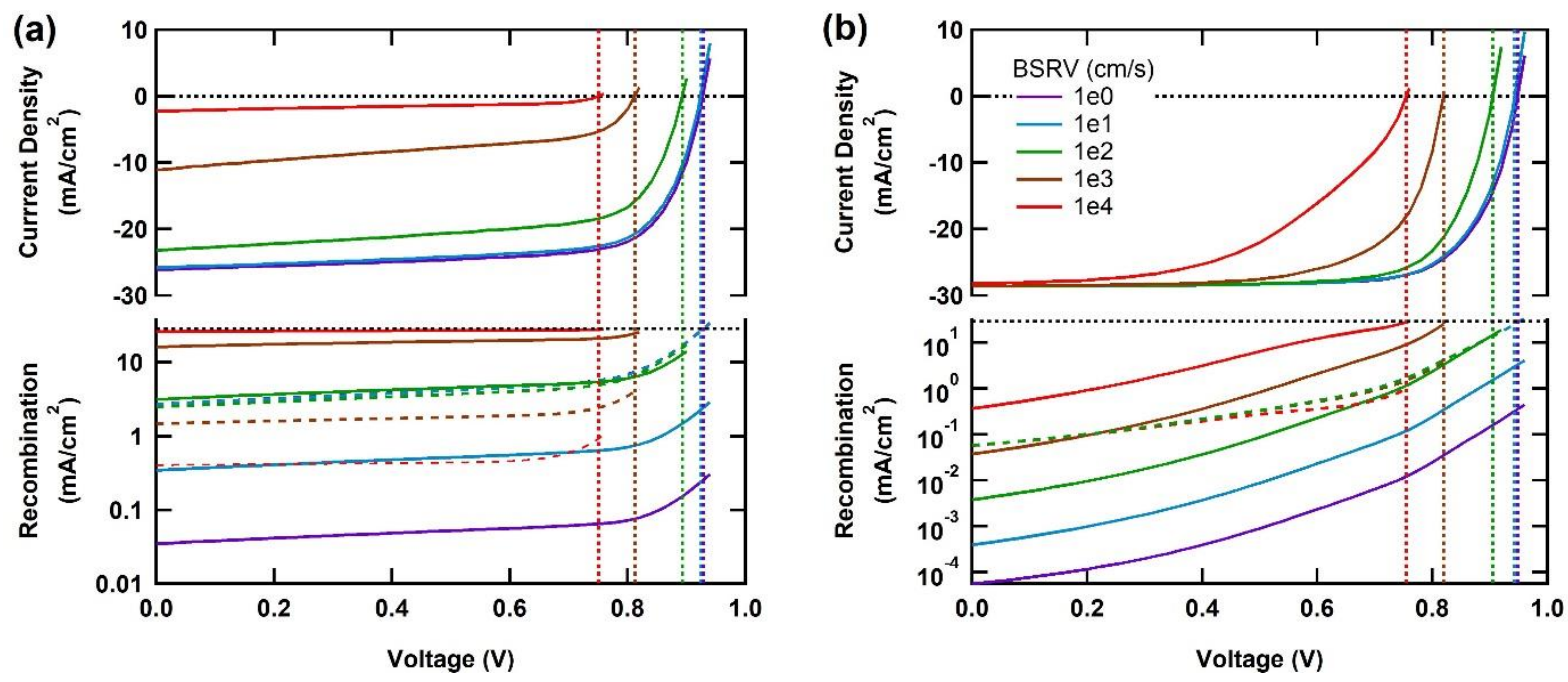

Fig. S3. Simulated current density-voltage and recombination current density-voltage curves for (a) $4 \mu \mathrm{m}$ and (b) $2 \mu \mathrm{m}$ absorber thickness for several back surface recombination velocities.

\section{Converting Charges in Back Buffer Layer to IFLO}

We employed a method similar to Kotipalli et al. to incorporate fixed charges into our model ${ }^{6}$, in which we include a $2 \mathrm{~nm}$ layer with the same properties as the absorber and vary the hole density to simulate the changes in charge density. The change in acceptor density shifts the Fermi level of this buffer layer providing an IFLO value and results in different band bending and electric field (E) at the back of the device. The fixed charge density in the film is related to the electric field by assuming the fixed charges create an infinite plane so $E=\sigma / 2 \varepsilon_{0}$ where $\sigma$ is the charge density and $\varepsilon_{0}$ is the permittivity of free space. Fig. S4 shows how the IFLO relates to the fixed charge density.

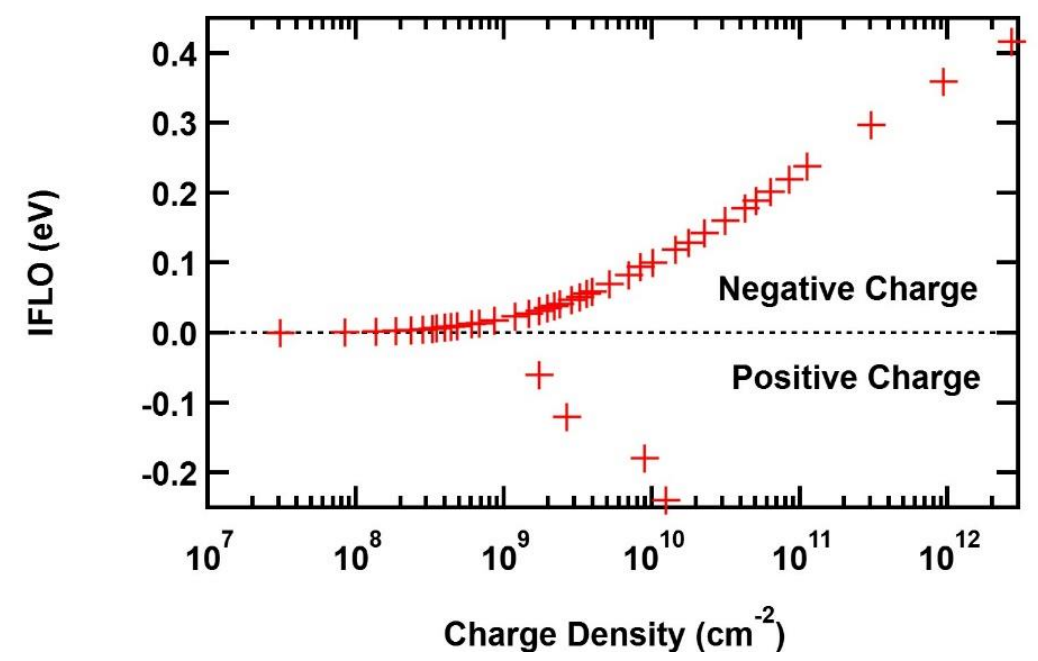

Fig. S4. IFLO value as a function of surface charge density. Above the dotted line indicates when there is negative charges at the interface, while below the line indicates when there are positive charges. 


\section{Effects of Initial Fermi Level Offset}

Figs. S5 and S6 show the PV parameters of CdTe devices as a function of IFLO and CdTe thickness for devices illuminated through the front of the device and the ratio of devices illuminated through the back to those illuminated through the front, respectively. These results provide a reference for device performance to compare with devices illuminated through the back of the device as reported in the main text. As opposed to back illuminated devices, thinner devices illuminated through the front with poor alignment at the back interface (IFLO $=-0.2 \mathrm{eV}$ ) have lower device performance.
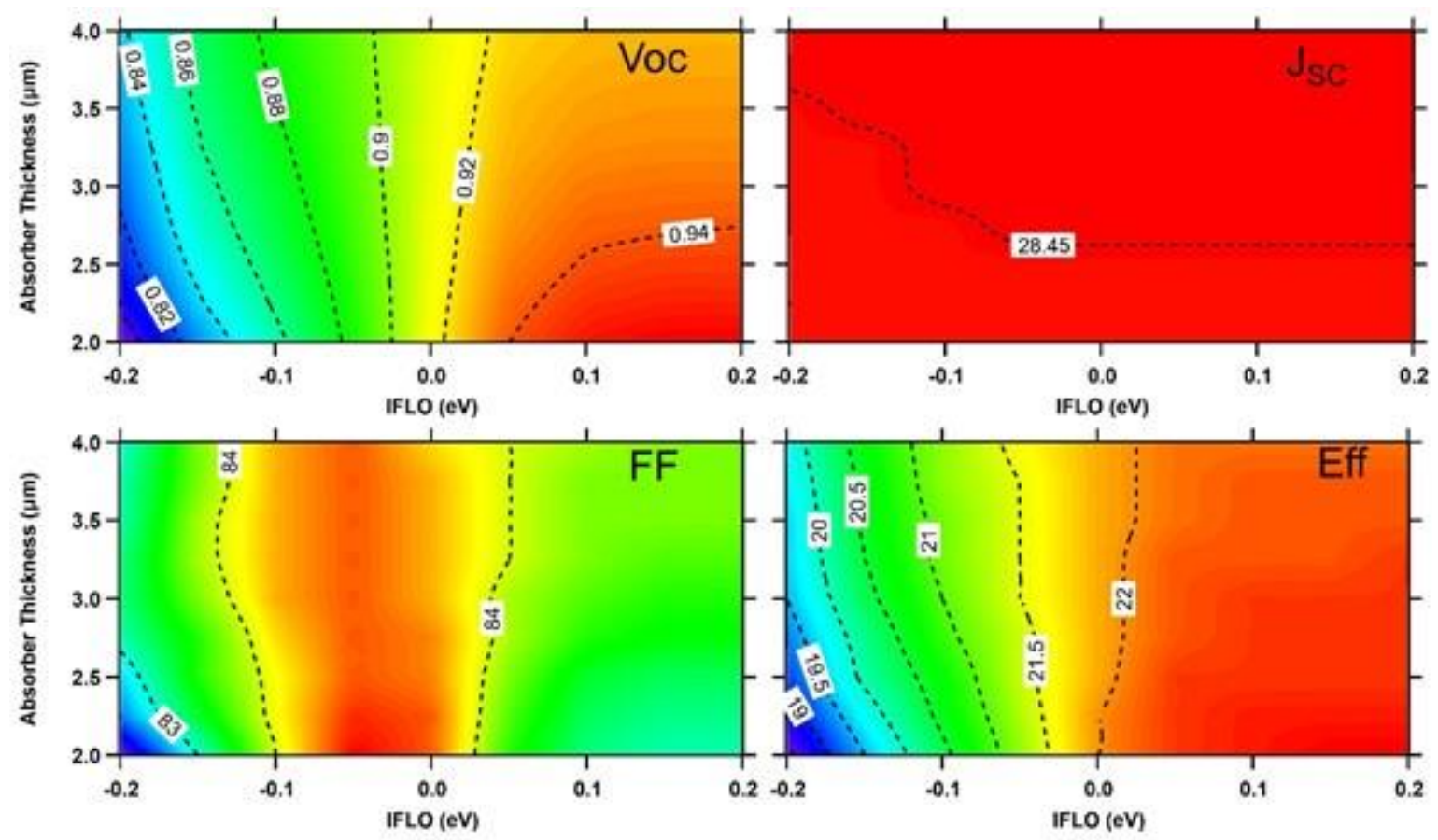

Fig. S5. Contour plots of open circuit voltage, short circuit current density, fill factor, and efficiency of CdTe devices illuminated through the front of the device as a function of initial Fermi level offset and absorber thickness. 


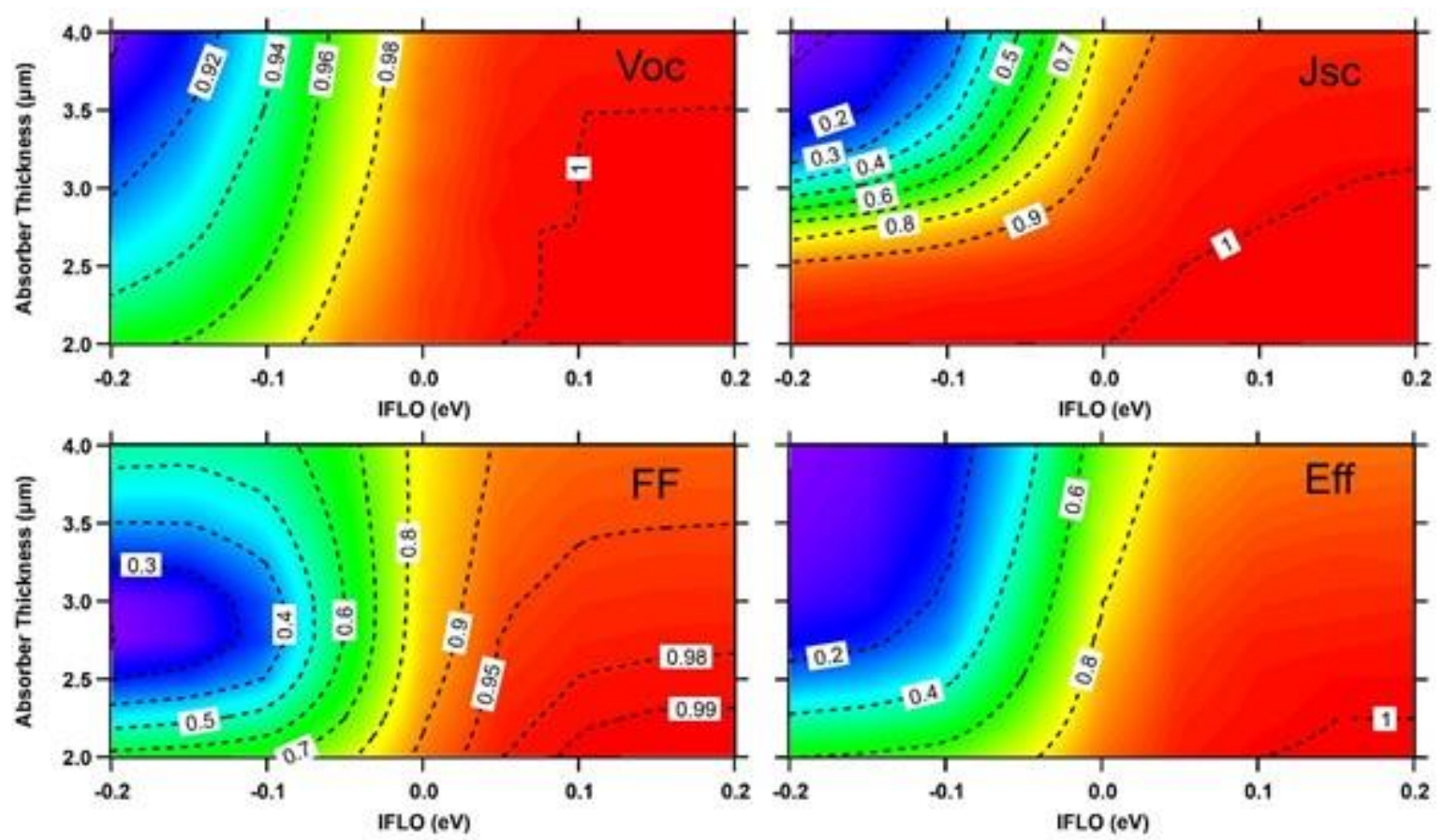

Fig. S6. Contour plots of the ratio of open circuit voltage, short circuit current density, fill factor, and efficiency of CdTe devices illuminated through the back to devices illuminated through the front as a function of initial Fermi level offset and absorber thickness.

The FF contour shown in Fig. 2 of the main text shows that the FF goes through a minimum as a function of absorber thickness when the IFLO is negative. This feature can be understood through the interaction between the $\mathrm{FJ}$ and $\mathrm{BJ}$ to form the equilibrated band diagram. As the absorber thickness increases from $2 \mu \mathrm{m}$, this interaction begins to decrease. Consequently, a depletion region at the back of the device begins to emerge at lower bias voltages, reducing the fill factor. At CdTe thickness of $\sim 2.75 \mu \mathrm{m}$, the FF reaches a minimum. This thickness also corresponds to a drop in the $\mathrm{J}_{\mathrm{Sc}}$, which, as shown in Fig. S7, is when the $\mathrm{BJ}$ depletion regions begins to emerge in the band diagram at zero bias. 


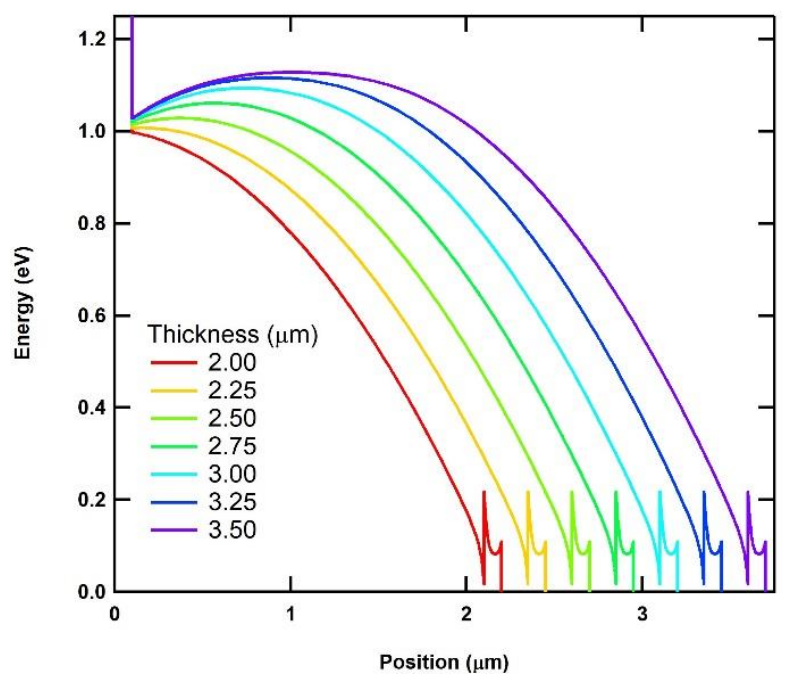

Fig. S7. Conduction band of devices for varying CdTe thicknesses at short circuit.

\section{Effect of Absorber Hole Density and Carrier Lifetime on PV Parameters}

Fig. 88 shows the efficiency for 2 and $4 \mu \mathrm{m}$ devices when the back interface has positive IFLO and a BSRV of $1 \times 10^{1} \mathrm{~cm} / \mathrm{s}$. These results are nearly identical to the one shown in Fig. 3 of the main text when the back interface has positive IFLO and BSRV of $1 \times 10^{4} \mathrm{~cm} / \mathrm{s}$, indicating that the upward band bending greatly reduces the importance of BSRV.
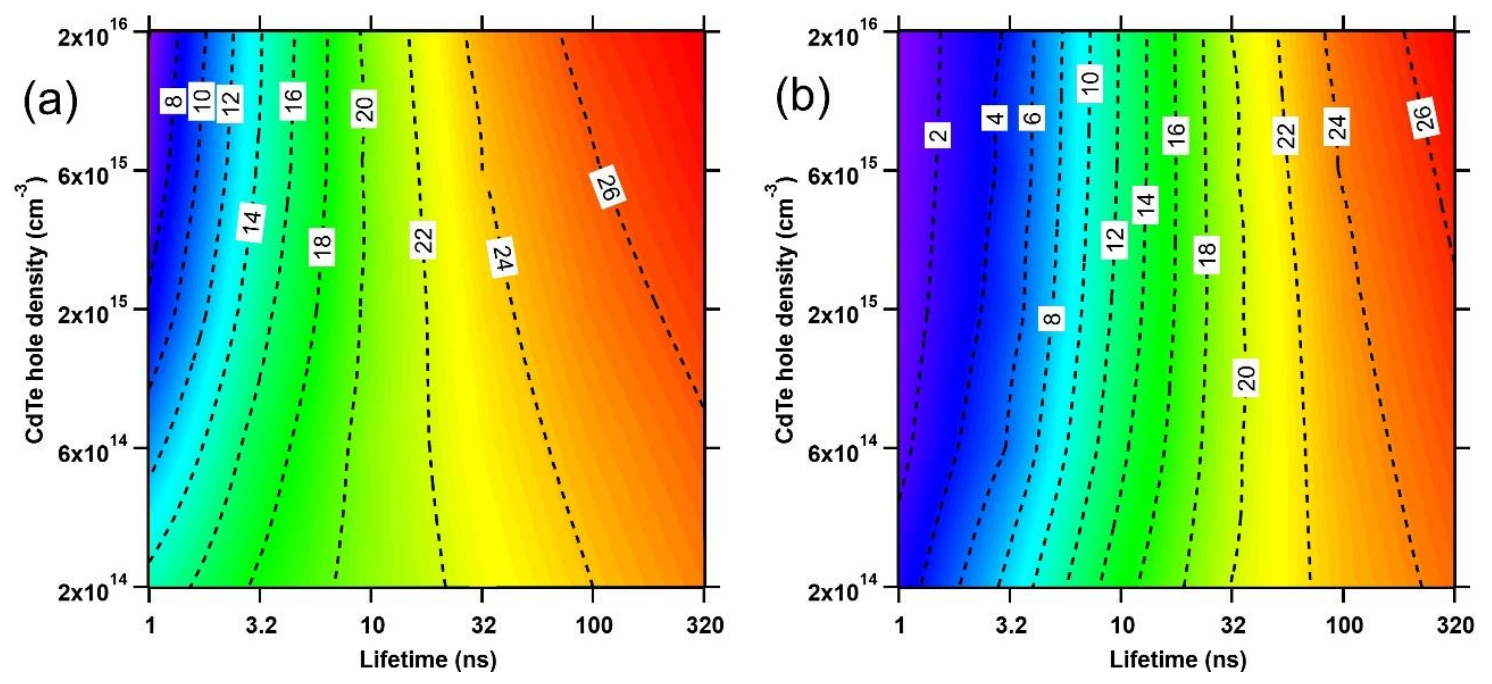

Fig. S8. Efficiency as a function of both CdTe hole density and carrier lifetime for devices with absorber thickness of (a) 2 and (b) $4 \mu \mathrm{m}$ when the IFLO is positive and the BSRV is $1 \times 10^{1} \mathrm{~cm} / \mathrm{s}$.

Figs. S9 and S10 show the PV parameters associated with the efficiency in Fig. 3 in the main text. 

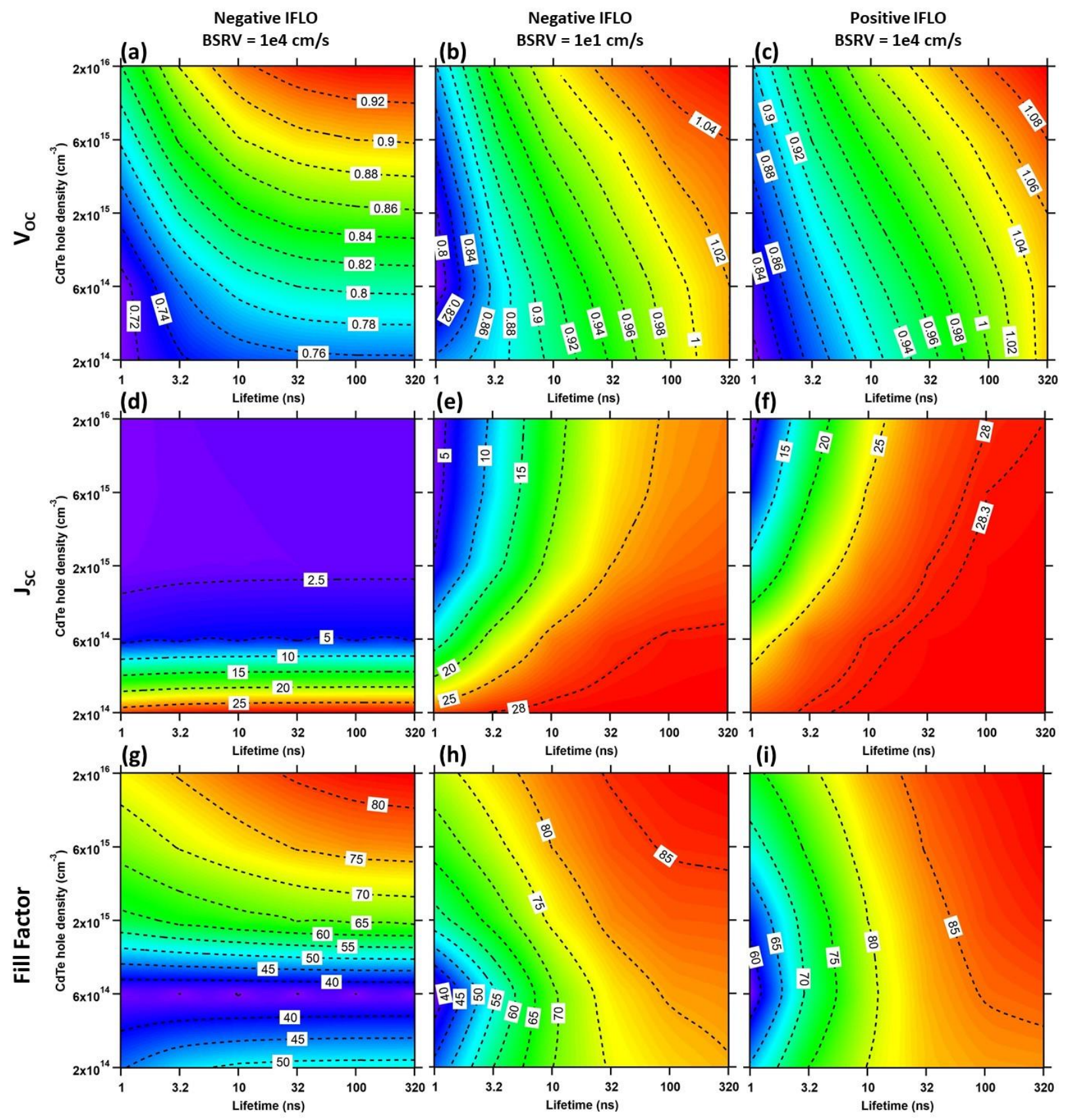

Fig. S9. Contour plots of the (a-c) $V_{o c}$, $(d-f) J_{s c}$, and (g-i) FF of a $2 \mu \mathrm{m}$ device illuminated through the back with $(a, d, g)$ negative IFLO and BSRV of $1 \times 10^{4} \mathrm{~cm} / \mathrm{s},(b, e, h)$ negative IFLO and BSRV of $1 \times 10^{1} \mathrm{~cm} / \mathrm{s}$, and $(c, f, i)$ positive IFLO and BSRV of $1 \times 10^{4} \mathrm{~cm} / \mathrm{s}$ the same conditions shown in Fig. $3 a, b$, and c, respectively, in the main text. 

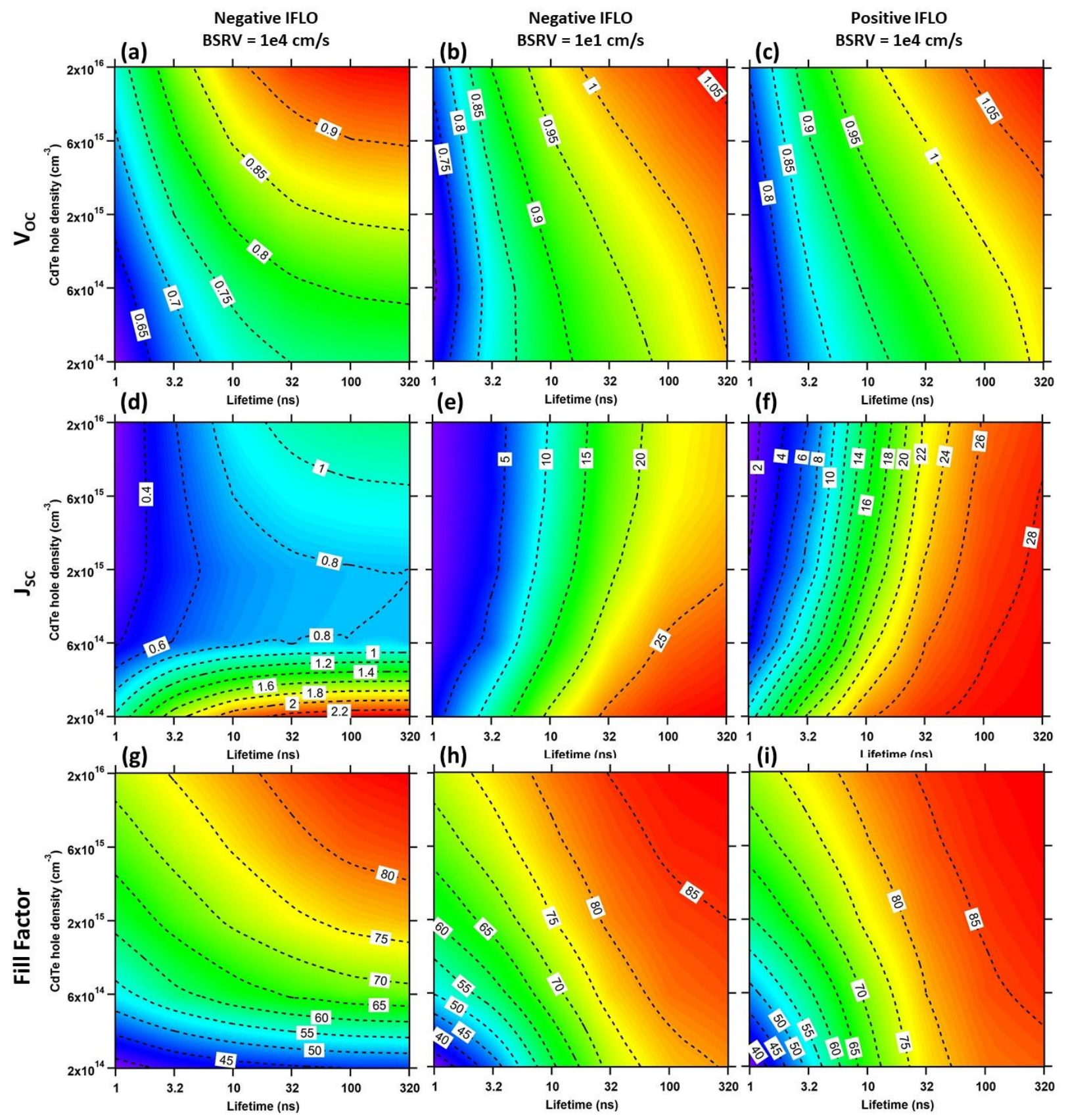

Fig. S10. Contour plots of the (a-c) $V_{o c},(d-f) J_{S c}$, and (g-i) FF of a $4 \mu \mathrm{m}$ device illuminated through the back with $(a, d, g)$ negative IFLO and BSRV of $1 \times 10^{4} \mathrm{~cm} / \mathrm{s},(b, e, h)$ negative IFLO and BSRV of $1 \times 10^{1} \mathrm{~cm} / \mathrm{s}$, and $(c, f, i)$ positive IFLO and BSRV of $1 \times 10^{4} \mathrm{~cm} / \mathrm{s}$ the same conditions shown in Fig. 3d,e,and $f$, respectively, in the main text. 

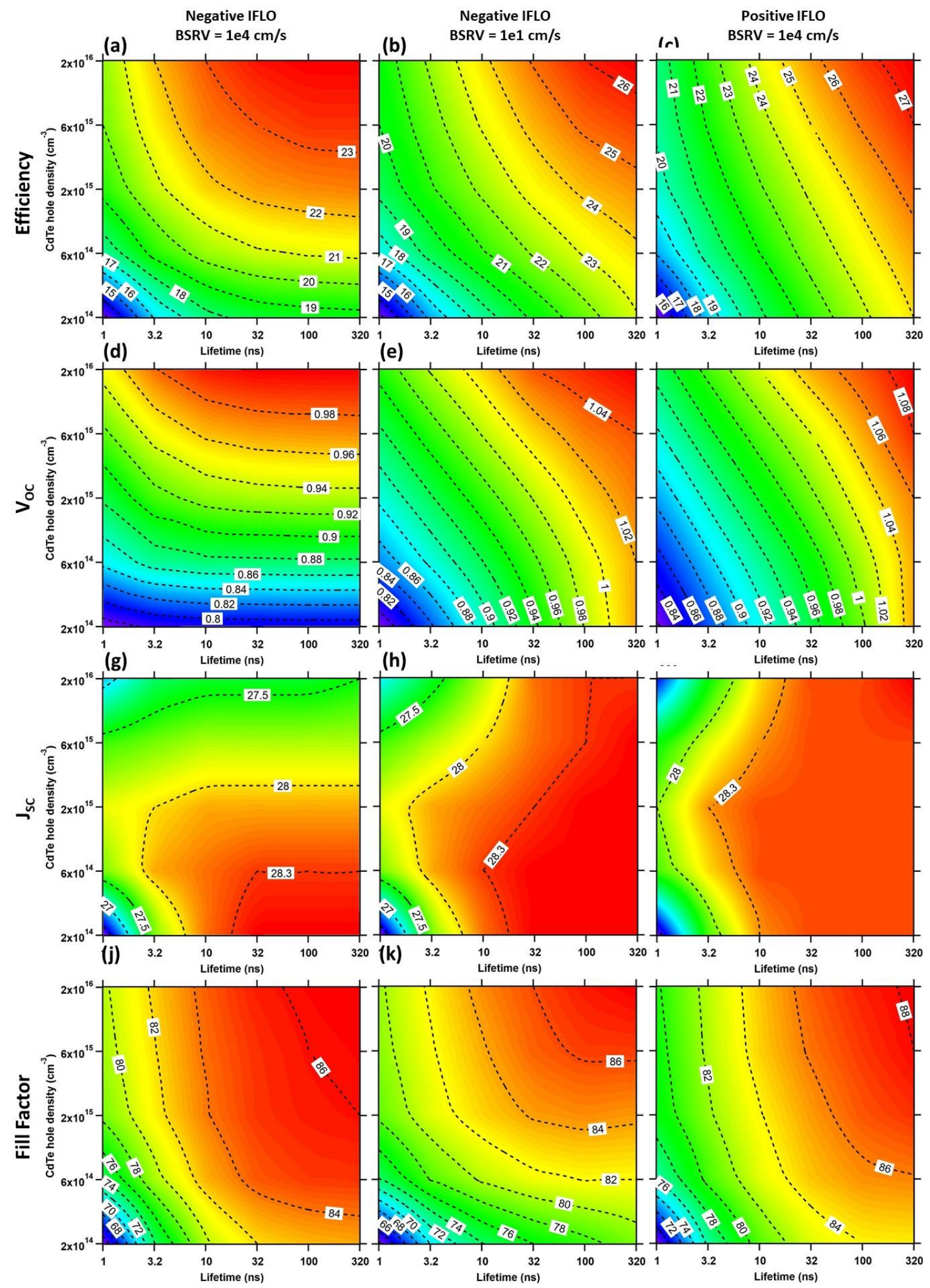

Fig. S11. Contour plots of the (a-c) efficiency, $(d-f) V_{O c},(g-i) J_{S c}$, and (j-I) FF of a $2 \mu m$ device illuminated through the front with $(a, d, g, j)$ negative IFLO and BSRV of $1 \times 10^{4} \mathrm{~cm} / \mathrm{s},(b, e, h, k)$ negative IFLO and BSRV of $1 \times 10^{1} \mathrm{~cm} / \mathrm{s}$, and $(\mathrm{c}, \mathrm{f}, \mathrm{I}, \mathrm{I})$ positive IFLO and BSRV of $1 \times 10^{4} \mathrm{~cm} / \mathrm{s}$. 
Supporting Information
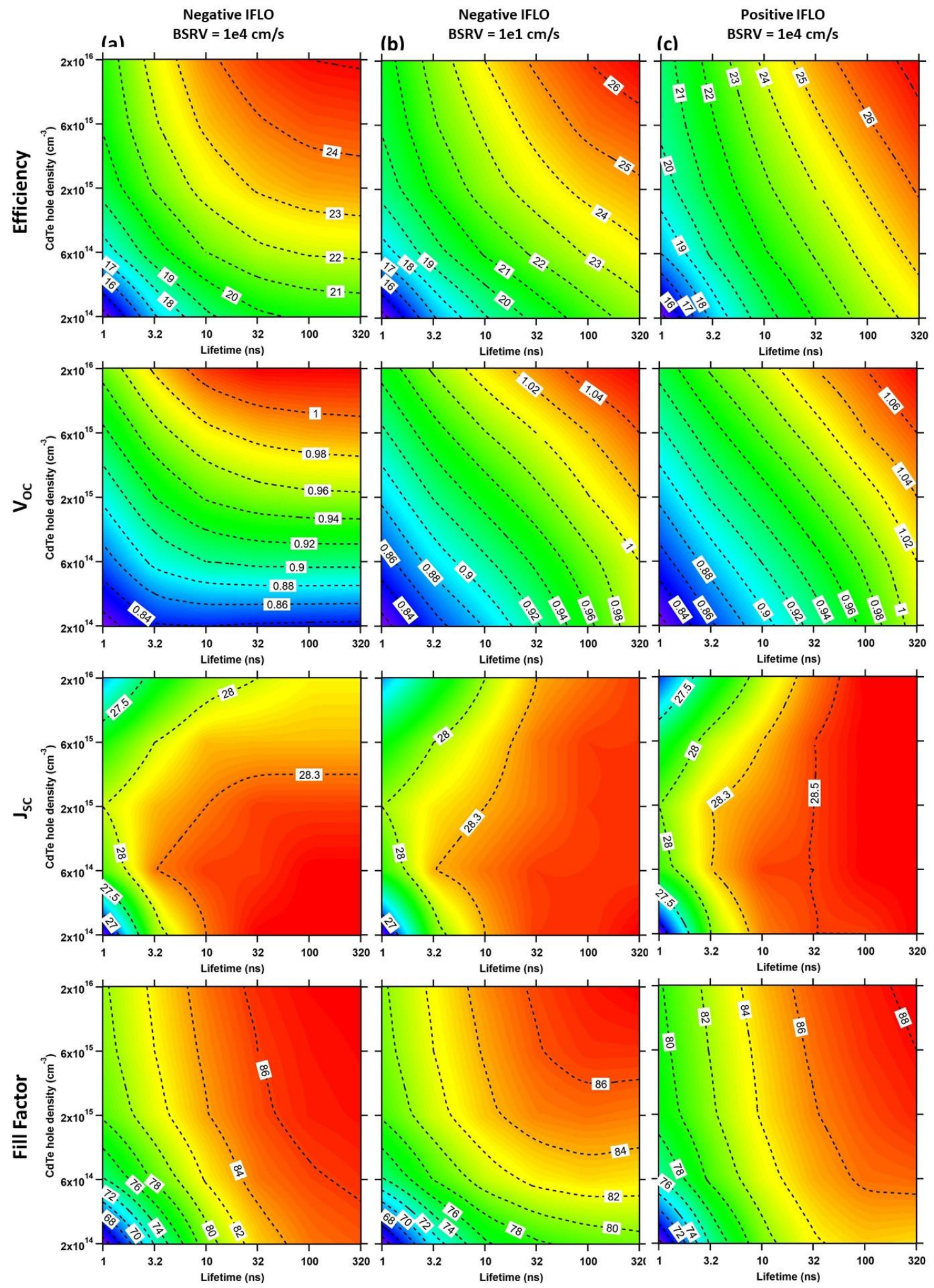

Fig. S12. Contour plots of the (a-c) efficiency, ( $d-f) V_{o c},(g-i) J_{s c}$, and $(j-I) F F$ of a $4 \mu m$ device illuminated through the front with $(a, d, g, j)$ negative IFLO and BSRV of $1 \times 10^{4} \mathrm{~cm} / \mathrm{s},(b, e, h, k)$ negative IFLO and BSRV of $1 \times 10^{1} \mathrm{~cm} / \mathrm{s}$, and $(\mathrm{c}, \mathrm{f}, \mathrm{I}, \mathrm{I})$ positive IFLO and BSRV of $1 \times 10^{4} \mathrm{~cm} / \mathrm{s}$. 


\section{Effects of Increased CdTe Dopant Density}

The band diagrams of the devices with $4 \mu \mathrm{m}$ and $2 \mu \mathrm{m}$ CdTe absorbers for three absorber dopant densities are shown in Fig. S13. This figure clearly shows that the depletion width at both junctions decreases with increased dopant density. In the case of the $2 \mu \mathrm{m}$ device, the increase in dopant density results in a BJ depletion width that is not present when the dopant density is low. As a result, the illuminated interface becomes more important, and in the case of backside illuminated devices, recombination increases. Fig. S14 shows the J-V and $\mathrm{J}_{\mathrm{r}}-\mathrm{V}$ of curves for these devices.
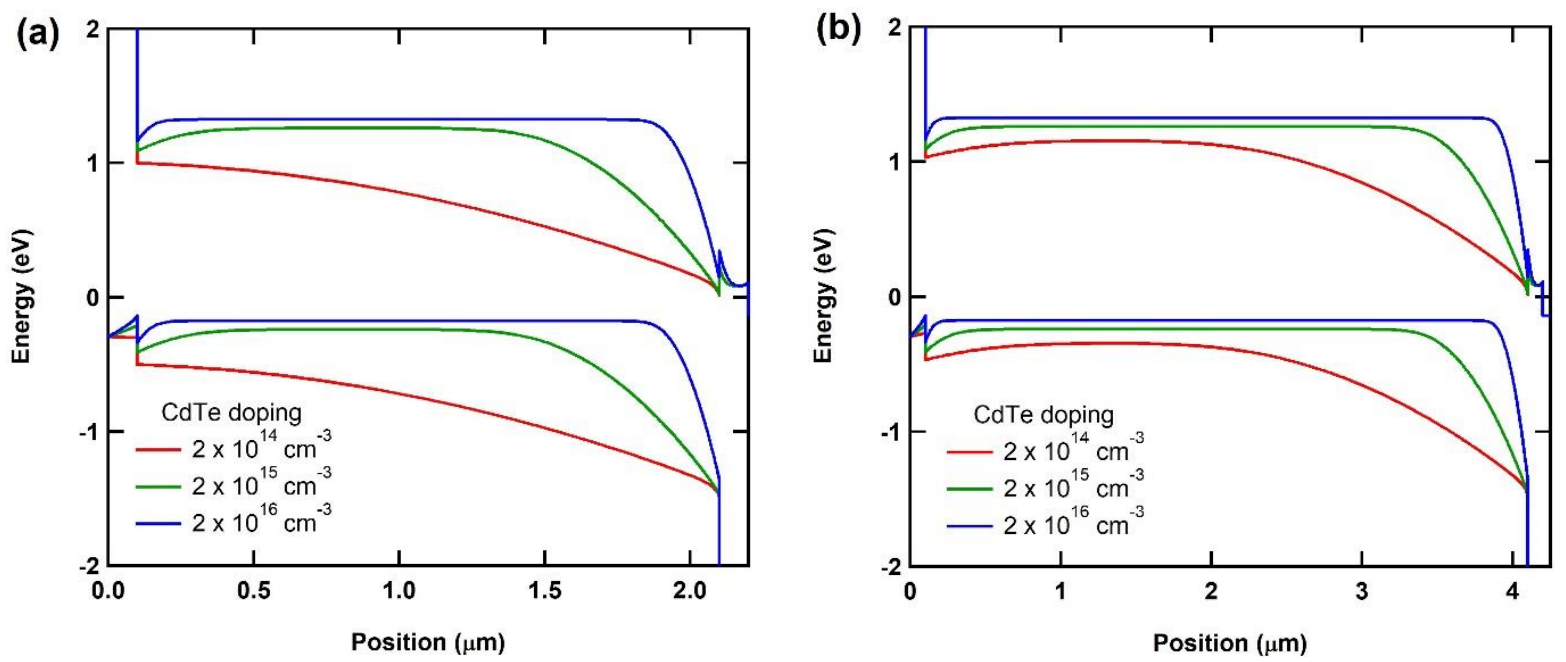

Fig. S13. Equilibrated band diagrams of CdTe devices with (a) $2 \mu \mathrm{m}$ and (b) $4 \mu \mathrm{m}$ absorber thickness for several absorber dopant densities at short circuit.
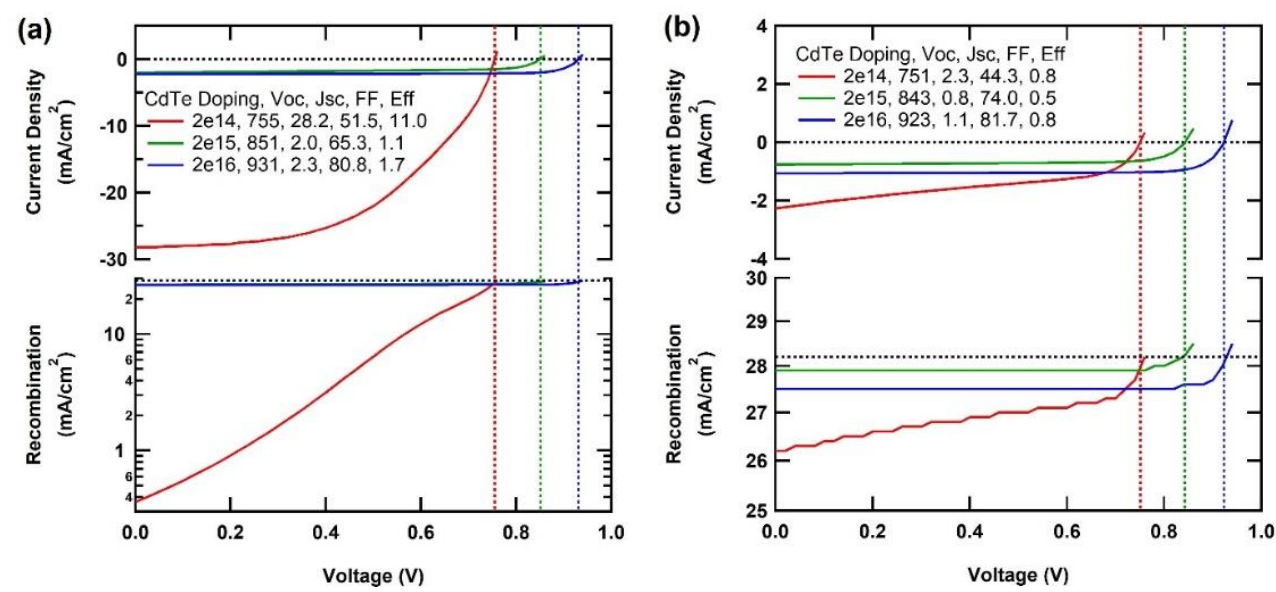

Fig. S14. Backside J-V and J $\mathrm{J}_{\mathrm{r}} \mathrm{V}$ for devise with absorber layer thickness of (a) $2 \mu \mathrm{m}$ and (b) $4 \mu \mathrm{m}$ for varying absorber dopant densities under one sun. Interface recombination remains orders of magnitude higher than Shockley-Reed-Hall recombination (not shown in these graphs). 
1. Gloeckler, M.; Fahrenbruch, A.; Sites, J. In Numerical modeling of CIGS and CdTe solar cells: setting the baseline, 3rd World Conference onPhotovoltaic Energy Conversion, 2003. Proceedings of, IEEE: 2003; pp 491-494.

2. Song, T.; Moore, A.; Sites, J. R., Te layer to reduce the CdTe back-contact barrier. Journal of Photovoltaics 2017, 8 (1), 293-298.

3. Song, T.; Kanevce, A.; Sites, J. R., Emitter/absorber interface of CdTe solar cells. Journal of Applied Physics 2016, 119 (23), 233104.

4. Liyanage, G. K.; Phillips, A. B.; Heben, M. J., Role of band alignment at the transparent front contact/emitter interface in the performance of wide bandgap thin film solar cells. APL Materials 2018, $6(10), 101104$.

5. Liyanage, G. K.; Phillips, A. B.; Alfadhili, F. K.; Ellingson, R. J.; Heben, M. J., The role of back buffer layers and absorber properties for $>25 \%$ efficient CdTe solar cells. ACS Applied Energy Materials 2019, 2 (8), 5419-5426.

6. $\quad$ Kotipalli, R.; Poncelet, O.; Li, G.; Zeng, Y.; Francis, L.; Vermang, B.; Flandre, D. J. S. E., Addressing the impact of rear surface passivation mechanisms on ultra-thin $\mathrm{Cu}(\mathrm{In}, \mathrm{Ga}) \mathrm{Se}_{2}$ solar cell performances using SCAPS 1-D model. Solar Energy 2017, 157, 603-613. 